\title{
Reviewer Acknowledgements for Global Journal of Health Science, Vol. 8, No. 1
}

Global Journal of Health Science wishes to acknowledge the following individuals for their assistance with peer review of manuscripts for this issue. Their help and contributions in maintaining the quality of the journal is greatly appreciated.

Global Journal of Health Science is recruiting reviewers for the journal. If you are interested in becoming a reviewer, we welcome you to join us. Please find the application form and details at http://www.ccsenet.org/reviewer and e-mail the completed application form to gjhs@ccsenet.org.

\section{Reviewers for Volume 8, Number 1}

Aljameel Albandary
Ama Pokuaa Fenny
Anjan Ghosh
Asad Ali Khan Afridi
Ashwini Kucknoor
Azizollah Arbabisarjou
Azizollah Arbabisarjou
Bruria Adini
Daleep Kumar Arora
Dalia Salah El-Deen El-Sedawy
Daniel Weber
David Walwyn
Dongxu Fu
Enrico Oddone
Evangelia Mavrikaki
Evanthia Sakellari
Francisco Rodenas
Gabriele Messina
Helen L. Smits
Isabela Mateus Martins
Jason Tsai
Jose C. Cordon
Kartheek Balapala
Katarina Paunovic
Kinley Wangdi
Liu Daws

Madhu Ouseph

Manju Sharma

Mansoor Ali Syed

Maria Malliarou

Martin Edwards

Masitah Shahrill

Montarat Thavorncharoensap

Nima Hafezi Nejad

Pavlos Sarafis

Pelechas Eleftherios

Polly Yeung

Preeti Viswanathan

Radu-Iulian Spataru

Raildo Coqueiro

Robert Sloan

Samendra Sherchan

Sara Melo

Satoshi Horiuchi

Shuqi Wang

Soon Yeng Soo Hoo

Sreenivas Phani Veeranki

Srikrishna Ramachandra

Tamilselvi Rajendran

Trisha Dunning

Vasudha Bansal

Wenhua Lu

William Cho

Yasam Kemal Akpak 